DIGITALCOMMONS - @WAYNESTATE -
Clinical Research in Practice: The Journal of Team Hippocrates

Volume 5 | Issue 1

Article 7

2019

\title{
NPO or AMA? Patients allowed clear liquids up to 2 hours pre-procedure have equivalent aspiration risk and increased patient satisfaction
}

Erich Smith

University of Wayne State Medical School, ersmith@med.wayne.edu

Follow this and additional works at: https://digitalcommons.wayne.edu/crp

Part of the Anesthesia and Analgesia Commons, Medical Education Commons, Surgical Procedures, Operative Commons, and the Translational Medical Research Commons

\section{Recommended Citation}

SMITH E. NPO or AMA? Patients allowed clear liquids up to 2 hours pre-procedure have equivalent aspiration risk and increased patient satisfaction. Clin. Res. Prac. 2019 Feb 6;5(1):eP1767. doi: 10.22237/crp/1549411680

This Critical Analysis is brought to you for free and open access by the Open Access Journals at DigitalCommons@WayneState. It has been accepted for inclusion in Clinical Research in Practice: The Journal of Team Hippocrates by an authorized editor of DigitalCommons@WayneState. 


\title{
NPO or AMA? Patients allowed clear liquids up to 2 hours pre-procedure have equivalent aspiration risk and increased patient satisfaction
}

ERICH SMITH, B.A., Wayne State University School of Medicine, ersmith@med.wayne.edu

\begin{abstract}
A critical appraisal and clinical application of Itou K, Fukuyama T, Sasabuchi Y, et al. Safety and efficacy of oral rehydration therapy until $2 \mathrm{~h}$ before surgery: a multicenter randomized controlled trial. Journal of Anesthesia. 2012;26(1):20-27. doi: $10.1007 / \mathrm{s} 00540-011-1261-x$.
\end{abstract}

Keywords: $\quad$ NPO, nil per os, preoperative fasting, fasting, aspiration

\section{Clinical Context}

Mr. J.P. was a 61-year-old obese male (BMI 40) with a history of non-alcoholic steatohepatitis cirrhosis, atrial fibrillation, hypertension, and type 2 diabetes. He presented to his primary care doctor with complaints of four days of disorientation, decreased appetite, fatigue, and dysuria and was referred to the Emergency Room. In the emergency department, he was found to have a urinary tract infection and anemia with a hemoglobin of $7.5 \mathrm{mg} / \mathrm{dl}$ with an unknown baseline. He provided a hard copy of his most recent esophagogastroduodenoscopy (EGD), which showed gastric varices. Because of this, he was suspected to have a GI bleed. He was treated appropriately with antibiotics, fluids, and a pantoprazole drip. A rectal exam and a CT abdomen pelvis were negative for any bleeding, and he was kept nil per os (NPO) for an EGD the next day. During the stay, the patient remained NPO and stable overnight. He became frustrated by his NPO status and called the attending on his cell-phone that had been given to him on a business card. He had threatened to leave against medical advice because of his NPO status but was successfully convinced to stay. He was classified as physical status American Society of Anesthesiologists (ASA) III. This classification is a designation of the patient's fitness level before a surgical procedure. There are six classes, and a designation of class III means that a patient has severe systemic disease. The patient had an EGD the next day that revealed no esophageal varices, and isolated type 1 gastric varices without bleeding or any recent stigmata of bleeding. The patient remained stable and was discharged with close PCP and GI follow up. The patient's frustration with his NPO status invites assessment of the utility of prolonged NPO status for procedures, based on the current evidence for pre-procedural NPO status.

\section{Clinical Question}

Does keeping patients NPO at midnight the night before a procedure offset the risk of aspiration with enough significance, when compared to clear liquids up to 2 hours before a procedure, to justify potential patient dissatisfaction?

ERICH SMITH, B.A., is a medical student at Wayne State University School of Medicine. 
SMITH E. NPO or AMA? Patients allowed clear liquids up to 2 hours pre-procedure have equivalent aspiration risk and increased patient satisfaction. Clin. Res. Prac. 2019 Feb 6;5(1):eP1767. doi: $10.22237 / \mathrm{crp} / 1549411680$
Clinical Research in Practice The Journal of Team Hippocrates

VOL 5 ISS 1 / eP1767 / FEBRUARY 6, 2019 doi: $\underline{10.22237 / \mathrm{crp} / 1549411680}$

\section{Research Article}

Itou K, Fukuyama T, Sasabuchi Y, et al. Safety and efficacy of oral rehydration therapy until $2 \mathrm{~h}$ before surgery: a multicenter randomized controlled trial. Journal of Anesthesia. 2012;26(1):20-27. doi: 10.1007/s00540-011-1261-x

\section{Related Literature}

The review of the literature began with a PubMed search using "nil per os" which returned 180 articles, and "NPO" which returned approximately 1,000 articles. The titles and abstracts of these results revealed that these articles either explored having a patient NPO during a specific clinical situation, such as bowel obstruction or pancreatitis, or were completely unrelated to the desired topic. A revised search using "preoperative fasting" returned 1,600 articles that were most relevant to the clinical situation. These search criteria resulted in articles that dealt with the practice of fasting before a procedure. A top recommended result was the practice guidelines for preoperative fasting from the Journal of the American Society of Anesthesiologists. A look at the American Society of Anesthesiology guidelines states that healthy patients can consume clear liquids up to two hours before surgery..$^{1}$ All major anesthesia organizations and societies recommend that clear liquids should be stopped two hours prior to a procedure based on the known physiology of gastric emptying. The two-hour cutoff is not because of experimental evidence. These practice guidelines cited many relevant historical and contemporary studies. One of these early studies, conducted by Suzuki and colleagues, occurred in 1996 in Japan. Suzuki et al. did not find a significant difference in gastric fluid volume in patients kept NPO from 9PM the day before surgery and patients who did not eat solid foods from 9PM but were allowed to drink clear liquids until 2 hours before anesthesia. $\underline{2}$ This study did not seek to answer the question of how the patient's emotional status was affected by either being NPO or allowed to drink liquids. A meta-analysis of studies published between 1950 and 2009 was conducted by the European Society of Anaesthesiology and resulted in published guidelines that adults and children should be encouraged to drink clear liquids up to 2 hours before elective surgery and that solid foods should be restricted for 6 hours.. A single-blind randomized study was conducted in 2004 by Maltby et al. in patients undergoing elective surgery with ASA physical status of I or II and BMI > 30 which found no statistical difference in gastric fluid volume immediately after anesthesia induction in patients who drank $300 \mathrm{ml}$ of clear liquid 2 hours before surgery versus those who did not, concluding that obese patients without other comorbidities should follow the same guidelines for preoperative fasting. .4

Summarizing all of the research trials shows agreement that drinking clear fluids up to 2 hours before an elective surgery in low-risk and obese patients is deemed safe, due to the insignificant difference in gastric fluid volume and subsequent theoretical risk of aspiration, when compared to keeping a patient NPO at midnight. However, there are shortcomings to all of these studies, and a meta-analysis Cochrane review was conducted in 2003 by Brady et al. that reviewed 22 randomized controlled trials and revealed that the studies involved healthy adults who were not at baseline increased risk of aspiration and that the studies did not report aspiration as an outcome. $\frac{5}{-}$ They instead relied on a theoretic risk of aspiration based on gastric volume content..$\frac{5}{\text { However, this }}$ review also found that there was no significant difference between gastric content between patients on a strict fast versus those who were permitted to drink clear liquids up to 2 hours before surgery. ${ }^{5}$ The Cochrane review was analyzed for any additional relevant citations.

Despite this research, it is the practice of many hospital systems to restrict food and liquids completely from midnight before a procedure requiring general anesthesia. A recent study by Itou et al. looked at the safety and efficacy of oral hydration up to 2 hours before surgery. ${ }^{-}$This study was deemed to be superior to previous studies because it had a large number of patients, and examined variables that imply theoretical aspiration risk, actual aspiration events, physiological differences between groups, and patient satisfaction. The most unique and important aspect of this study is that it assessed how patient's emotions, sensations, and satisfaction were affected by fasting, making it especially clinically relevant to our patient This study was critically appraised to review these recommendations, and it is discussed in the next section.

\section{Critical Appraisal}

Itou et al., based in Japan, performed a multicenter randomized control trial involving 6 university-affiliated hospitals. $\underline{6}$ Three hundred low-risk patients that were scheduled for a morning surgery were computer randomized with a permuted-block design to an oral rehydration solution (ORS) group or to a fasting group. These low-risk patients were between 20 and 80 years old, having 
SMITH E. NPO or AMA? Patients allowed clear liquids up to 2 hours pre-procedure have equivalent aspiration risk and increased patient satisfaction. Clin. Res. Prac. 2019 Feb 6;5(1):eP1767. doi: $10.22237 / \mathrm{crp} / 1549411680$
Clinical Research in Practice The Journal of Team Hippocrates

VOL 5 ISS 1 / eP1767 / FEBRUARY 6, 2019 doi: $\underline{10.22237 / \mathrm{crp} / 1549411680}$

elective surgery under general anesthesia, and were ASA physical status I or II. The two randomized groups were similar at baseline. This trial, like previous studies, had the shortcoming of only including low-risk patients. Exclusion criteria included age over 80 , BMI greater than $35 \mathrm{~kg} / \mathrm{m}^{2}$, pregnant women, and patients with ASA III or higher. The patient in this clinical scenario would be excluded from this study based on his BMI of 40 and ASA III status. However, previous studies have shown that obese patients do not have increased risk of aspiration, and patients with ASA status greater than II have never been included in any randomized control study. The ORS contained glucose and electrolytes, and the ORS group was able to consume 1,000 ml divided into $500 \mathrm{ml}$ from 9 pm until they went to sleep and $500 \mathrm{ml}$ from the time they woke up until 2 hours before surgery. The fasting group was placed NPO from 9 pm the night before surgery. $\underline{6}$

Primary endpoints included gastric content volume and $\mathrm{pH}$ measured after anesthesia induction. Secondary endpoints included whether or not there was vomiting, aspiration, correlation between BMI, age, and gastric volume, vital signs, and importantly patient comfort. With regards to patient comfort it is important to note that this was not a double-blinded study. Patients were aware they were part of a group that was able to drink liquid up until 2 hours per surgery and then were asked to fill out a questionnaire after the procedure, which could instill a response bias. $\underline{6}$

The statistical methods for this study were strong. The null hypothesis was set that there was no difference between gastric volumes in the study groups. Significance level was 0.05 with a power of 0.8 necessitating a group size of 122 . The sample size per group was then increased to 150 for a total of 300 patients. $\underline{6}$

Both groups had similar patient withdrawal from the study due to reasons such as revoked consent and cancelled procedures. A total of 26 patients withdrew from the study, which left enough patients in both groups to keep the threshold above 122 . The recorded volumes of fluid consumed by the ORS group was sufficient with the average patient drinking $414 \mathrm{ml}$ the night before and $398 \mathrm{ml}$ the morning of surgery. This demonstrates the seemingly obvious point that if patients are allowed to drink before surgery, they will.

The study showed that there was no significant difference $(P=0.3)$ in gastric volume at induction between the ORS group and fasting group. Gastric $\mathrm{pH}$ also did not have a significant difference $(\mathrm{P}=0.59)$. There were no instances of vomiting or aspiration in either group, which is not surprising since all of the patients were selected to be low risk. No significant correlation was found between the $\mathrm{BMI}$ and gastric fluid volume $(\mathrm{P}=0.85)$. However, in the ORS group, there was a significant inverse correlation between age and gastric fluid volume $(P=0.001) . \underline{6}$

Reported thirst and hunger in the ORS group was significantly lower than in the fasting group $(P<0.001)$ without a significant difference in reporting of anxiety, nausea, or vomiting. This important aspect of the research is the most clinically relevant. It has been well established in past literature that there is no increased risk of aspiration, but this finding suggests a positive outcome that would directly affect patient satisfaction.

This multicenter randomized control trial meets Category A and level 1 study quality with the SORT criteria. $\underline{7}$

Limitations of this study are the exclusion of patients who are not ASA I or II. Patients recruited for the study were patients who did not have a high risk of aspiration. These patients were in fact excluded. This study leaves out a significant patient population including the patient mentioned in the clinical summary. The use of blinding in this study was not feasible. The staff of the hospital, the patient, and the physicians all knew if a patient was part of the study and what group they were in. Besides being given access to liquids, all of the patients were treated the same throughout their hospital stay. Randomization was adequate and accomplished via a computer. The simple intervention of providing an ORS used in this study would be feasible for any hospital.

\section{Clinical Application}

Our patient threatened to leave against medical advice (AMA) and contacted the attending after hours on a personal cell phone to express his displeasure in being NPO for a procedure. A major conclusion of Itou et al. is that patients who are given access to ORS have significantly less hunger and thirst during the period of time before a scheduled procedure, and experience no significant increase in negative outcomes. This study has external validity when applied to our patient. If he were given ORS, he would have had a significant outcome of being less thirsty and therefore less likely to threaten to leave AMA: a benefit were the research applied here. However, the 
SMITH E. NPO or AMA? Patients allowed clear liquids up to 2 hours pre-procedure have equivalent aspiration risk and increased patient satisfaction. Clin. Res. Prac. 2019 Feb 6;5(1):eP1767. doi: $10.22237 / \mathrm{crp} / 1549411680$
Clinical Research in Practice The Journal of Team Hippocrates

VOL 5 ISS 1 / eP1767 / FEBRUARY 6, 2019 doi: $10.22237 / \mathrm{crp} / 1549411680$

potential for negative outcomes in patients classified as ASA III has not been studied, which represents a potential harm and makes the study less applicable. Though the evidence is suggestive of a positive outcome, it is not definitive, and it would not be wise to justify that a patient with ASA III status be allowed to follow the same guidelines as an ASA I or II patient.

Learning points:

1. There is an abundance of research that shows that there is no significant increase in aspiration or vomiting in low risk patients who are allowed to drink clear liquids up to 2 hours before surgery.

2. Despite recommendations that patients should be restricted from solid foods for 6 hours pre-procedure, and clear liquids 2 hours pre-procedure, hospitals worldwide continue to make patients NPO at midnight.

3. Studies that have looked at the risk of aspiration and preoperative fasting have universally omitted patients who are high-risk for aspiration creating a selection bias that favors positive outcomes.

\section{References}

1. Practice guidelines for obstetric anesthesia: an updated report by the American Society of Anesthesiologists Task Force on Obstetric Anesthesia and the Society for Obstetric Anesthesia and Perinatology. Anesthesiology. Feb 2016;124(2):270-300. doi: 10.1097/ALN.0000000000000935

2. Suzuki A, Kumano H, Osaka S, Shiomi Y, Moroi K, Ishimura N, Nishiwada M. The effects of preoperative drinking and H2 blocker on gastric acid secretion (in Japanese with English abstract) Masui (Jpn J Anesthesiol) 1996;45:445-448.

3. Smith I, Kranke P, Murat I, Smith A, O'Sullivan G, Sreide E, Spies C, In't Veld B. Perioperative fasting in adults and children: guidelines from the European Society of Anaesthesiology. Eur J Anaesthesiol. 2011;28:556-569. doi: 10.1097/EJA.0b013e3283495ba1

4. Maltby JR, Pytka S, Watson NC, Cowan RA, Fick GH. Drinking $300 \mathrm{~mL}$ of clear fluid two hours before surgery has no effect on gastric fluid volume and $\mathrm{pH}$ in fasting and non-fasting obese patients. Can J Anaesth. 2004;51:111-115. doi: $10.1007 / B F 03018767$

5. Brady M, Kinn S, Stuart P, et al. Preoperative fasting for adults to prevent perioperative complications. Cochrane Database Syst Rev. 2003;4:CD004423. doi: 10.1002/14651858.cd004423

6. Itou K, Fukuyama T, Sasabuchi Y, et al. Safety and efficacy of oral rehydration therapy until $2 \mathrm{~h}$ before surgery: a multicenter randomized controlled trial. Journal of Anesthesia. 2012;26(1):20-27. doi: 10.1007/s00540-011-1261-x

7. Ebell MH, Siwek J, Weiss BD, Woolf SH, Susman J, Ewigman B, et al. Strength of recommendation taxonomy (SORT): a patientcentered approach to grading evidence in the medical literature. The Journal of the American Board of Family Medicine. 2004;17(1):59-67. doi: 10.3122/jabfm.17.1.59 